\title{
Assessing the Correlation Between Location and Size of Catastrophic Breakdown Events in High-K MIM Capacitors
}

\author{
J. Muñoz-Gorriz, D. Blachier, G. Reimbold, F. Campabadal, J. Suñé, S. Monaghan, K. Cherkaoui, \\ P. K. Hurley, E. Miranda
}

\begin{abstract}
The connection between the spatial location of catastrophic breakdown spots occurring in metal-insulator-metal capacitors with a high-permittivity dielectric film $\left(\mathrm{HfO}_{2}\right)$ and their respective sizes is investigated. Large area structures $\left(10^{4}-10^{5} \mu \mathrm{m}^{2}\right)$ are used for this correlation assessment since, for statistical considerations, a large number of spots in the same device is imperatively required. The application of ramped or constant voltage stress across the capacitor generates defects inside the dielectric that result in the formation of multiple failure sites. High power dissipation takes place locally leaving a permanent mark on the top electrode of the device. The set of marks constitutes a point pattern with attributes that can be analyzed from a statistical viewpoint. The correlation between the spot locations and their sizes is assessed through the mark correlation function and the method of reverse conditional moments. The study reveals that for severely damaged devices there exists a link between the spot location and size that leads to a short range departure from a complete spatial randomness (CSR) process. It is shown that the affected region around each failure site is actually larger than the visible area of the spot. A structural modification of the dielectric layer in the vicinity of the spot caused by the huge thermal effects occurring just before the microexplosion might be the reason behind this extension of the damage.
\end{abstract}

Index Terms - High-K, reliability, oxide breakdown, spatial statistics.

\section{INTRODUCTION}

$\mathrm{T}$ HE breakdown (BD) of the oxide layer in metal-insulatormetal (MIM) and metal-insulator-semiconductor (MIS) structures is an unwanted event that, depending on its magnitude, can affect the electrical properties of the devices to different extents [1]. In the vast majority of the studies devoted to this issue, the origin and consequences associated with the occurrence of a single failure event per device is investigated. Very often, the study of single BD events in MIS and MIM structures exclusively relies on electrical measurements. Depending on the magnitude of the BD event, the failure mode is classified as soft or hard breakdown [2], [3]. By applying

J. Muñoz-Gorriz, J. Suñe and E. Miranda are with the Departament d'Enginyeria Electrònica, Universitat Autònoma de Barcelona, 08193 Cerdanyola del Valles, Spain (e-mail corresponding author: jordi.munoz.gorriz@uab.cat).

D. Blachier and G. Reimbold are with CEA-LETI, Grenoble, France. ramped or constant current/voltage stress to a large number of devices it is possible to obtain statistical information on the BD strength or the time/injected charge-to-BD, which is frequently used in reliability analysis to qualify a given technology [4]. However, generation of multiple BD events $(N>10)$ in the same device is seldom investigated because, as the total leakage current increases, the actual potential drop across the device considerably decreases because of the series resistance effect. As a consequence, although the electrical stress becomes less effective in what concerns with the generation of new failure sites, the already created defects can evolve because of the thermal effects. In some cases, correlation effects among the BD spots were demonstrated to impact on the lifetime projection of MIS devices [5]. Here, we are interested in elucidating whether the location and size of the BD spots are to some extent interrelated. The BD phenomenon basically consists in the formation of a short or conductive filament $(\mathrm{CF})$ across the dielectric film that allows an uncontrolled flow of electrons between the top and bottom electrodes. According to the percolation theory of dielectric $\mathrm{BD}$, the $\mathrm{CF}$ arises as the result of a critical density of defects accumulated at certain regions of the oxide film [6], [7]. Once the CF is established, a huge amount of thermal energy is released due to Joule heating effects occurring in a tiny area. Under this circumstance, the temperature increase can be so large that the top metal electrode blows up producing an irreversible damage to the structure. This is sometimes called hard BD with lateral propagation [8]. The ultimate consequence of this process is a mark on the top electrode that can be easily detected through an optical microscope [9]. In large area devices, such as the ones investigated in this work, several BD spots of this kind appear, and which can be collectively treated as a point pattern.

In previous works, we showed that the methods of spatial statistics are suitable for investigating the distribution of BD spots in MIM and MIS structures [10]-[12]. In spatial statistics, estimators are directly calculated from the experimental data and are compared with the corresponding theoretical values for

F. Campabadal is with the Institut de Microelectrònica de Barcelona, IMBCNM, CSIC, Cerdanyola del Valles, Spain.

S. Monaghan, K. Cherkaoui and P.K. Hurley are with the Tyndall National Institute, Cork, Ireland. 
a Poisson or complete spatial randomness (CSR) process [13]. In principle, the distribution of $\mathrm{BD}$ spots on the top electrode of a device is expected to follow a Poisson process since the generation of defects inside the dielectric is completely random. However, deviations arising from the particularities of the fabrication process, local variations of the oxide permittivity value, or edge effects can occur. In general, each statistical estimator highlights some specific feature of the point distribution. Thus a complete understanding of the problem under investigation can only be achieved by assessing and comparing the results obtained with different estimators [10]. In the field of spatial analysis not only the location of the points is of interest but also the value of one or more attributes attached to each point of the pattern. In our case, the attribute to be analyzed is the size of the spots (a continuous attribute). To the best of our knowledge, the correlation between the BD spot locations in MIM devices and their sizes is explored for the first time. This requires the characterization of many failure events occurring in a single device with a high optical resolution. In a first approach, the location of the spots is analyzed using a second order statistical estimator such as the pair correlation function with the objective of testing the CSR hypothesis. In a second approach, the connection between the location and size of the spots is investigated through more sophisticated tools of spatial statistics analysis such as the mark correlation function and the method of reverse conditional moments. We also focus the attention on the regions around the spots since they exhibit remarkable features. This is investigated combining SEM, CAFM and infrared images of the generated spots. This paper is organized as follows: in Section II, the fabrication process of the devices under study is presented. The experimental conditions used for generating the spots are described as well as the equipment and methods considered for analyzing the damaged devices. In Section III, the procedure used for extracting the location and area of the spots from the optical images and videos is explained. The correlation between the size and location of the BD spots for square and circular area capacitors is investigated in Section IV. The long term consequences of the degradation process in our devices is also discussed. Finally, in Section V, the damage generated around the crater-like structure of a single spot is explored by combining finite-element simulations with thermal images. It is shown that the device region around the visible spot also experiments some kind of modification which in turn affects the occurrence of the new generated failure sites. In Section VI, the conclusions of this work are presented.

\section{EXPERIMENTAL DETAILS}

Circular and square $\mathrm{Pt} / \mathrm{HfO}_{2} / \mathrm{Pt}$ capacitors with areas ranging from $4 \cdot 10^{4}$ to $10^{6} \mu \mathrm{m}^{2}$ were investigated. The devices were fabricated on top of an n-type $\mathrm{Si}(100)$ substrate. First, a $200 \mathrm{~nm}$ thick $\mathrm{SiO}_{2}$ layer was grown to electrically isolate the substrate from the devices. Then, a $200 \mathrm{~nm}$ thick Pt layer was deposited by electron-beam evaporation and a $30 \mathrm{~nm}$ thick $\mathrm{HfO}_{2}$ layer was grown by Atomic Layer Deposition (ALD) technique using TEMAHf and $\mathrm{H}_{2} \mathrm{O}$ as precursors. The equipment used to apply the ALD technique was a Cambridge NanoTech Fiji ALD

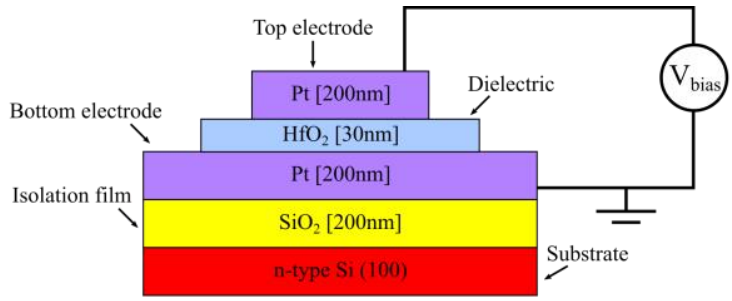

(a)

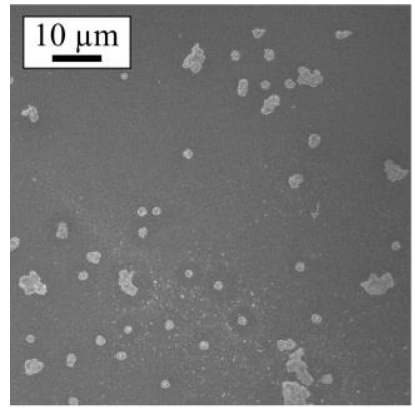

(b)

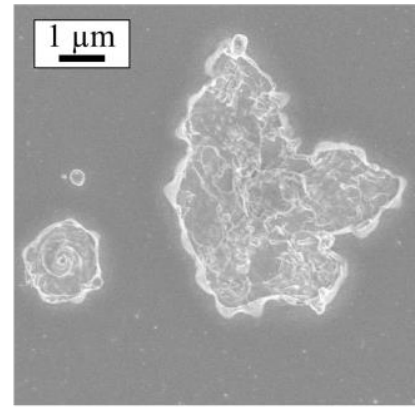

(c)
Fig. 1. (a) Schematic representation of the device structure and measurement setup. (b) SEM image of the BD spots generated over the top metal electrode after a voltage stress. (c) Zoomed-in SEM image of some BD spots.

system. Afterwards, a $200 \mathrm{~nm}$ thick Pt layer was deposited on the $\mathrm{HfO}_{2}$ layer using the lift-off technique allowing the definition of different top electrode geometries. Finally, the access to the bottom Pt electrode was enabled via the dry etching technique. A mask/resistor process was used to protect the top Pt electrode while the $\mathrm{HfO}_{2}$ layer was removed. After the etching, the oxide layer extends $25 \mu \mathrm{m}$ beyond the perimeter edge of the top electrode. During stress the bottom electrode was always grounded while the voltage was applied to the top electrode (see Fig. 1a). For the generation of BD spots, two different approaches were followed: constant voltage stress (CVS) at $-9 \mathrm{~V}$ for a maximum of 70 seconds and ramped voltage stress starting at $0 \mathrm{~V}$ and ending at $-12 \mathrm{~V}$. In the former mode, movies showing the evolution of the degradation process were recorded. In the second case, a picture with the final distribution of spots was taken. Depending on the magnitude, duration, kind of the stress (ramped or constant), and device area, different BD spot patterns can be obtained. Recall that this paper is not aimed at characterizing this particular technology but to provide a framework for the correlation analysis of multiple failure events in MIM and MIS devices. The location and size of the spots were obtained from videos and images recorded through the optical microscope. The information was subsequently processed with MATLAB and statistically treated using the Spatstat package for the R language [14].

Figure $1 \mathrm{~b}$ shows a scanning electron microscope (SEM) image of several BD spots generated on the top metal electrode of a device. Notice that the spots have a typical size ranging from 1 to $5 \mu \mathrm{m}$ and that their shape is not always circular. Circular shapes are mostly observed for the smallest spots. The image reveals that some of the spots exhibit a kind of ring around them. This will be further discussed in Section VI in connection with the thermal effects. In Fig. 1c, a zoomed-in 


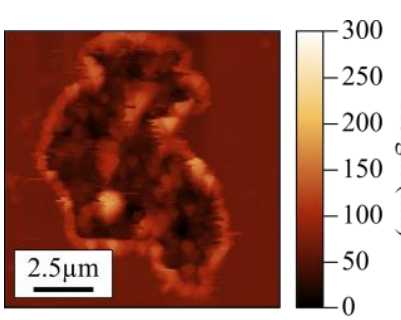

(a)

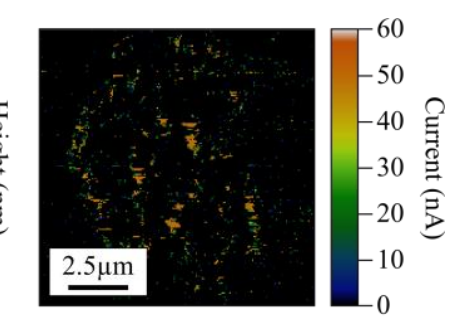

(b)

Fig. 2. (a) AFM image of a spot generated on the top electrode and (b) its corresponding conductive image.

SEM image of nearby BD spots is shown. As can be seen, the spots have a crater-like structure in which the top metal layer has practically disappeared. In order to get better insight whether the bottom metal electrode is accessible through the crater or not, topographic (Fig. 2a) and conductive (Fig. 2b) scannings were performed using a conductive atomic force microscope (CAFM). The conductive-type image (Fig. 2a) reveals that the current does not flow uniformly distributed and that the edges as well as some particular points inside the crater seem to exhibit a higher leakage current. It is worth mentioning that the current measured this way might be affected by the extreme topographic variations. According with a previous thermal study, some failure sites are true shorts while others do not exhibit any sign of electron transport [15]. In this paper, new infrared images are presented which indicate the magnitude of the power dissipation that takes place locally in the CF and the extension of the thermal damage around the BD spot.

\section{DETECTION OF SPOTS AND IMAGE TREATMENT}

In order to achieve accurate information about the spatial location and area of each failure site, a MATLAB program was developed for this aim. MATLAB has the capability to deal with images and counts with a large number of commands for data transformation, visualization, and analysis. When MATLAB opens an image file, three matrices with a number of rows and columns equal to the image resolution are created. Each matrix is associated with one of the basic colors. Some commands are specifically oriented to the determination of the spatial location and size of objects in the image (in our case the BD spots). In Fig. 3, the mathematical treatment performed on a typical image is recreated. Figure 3 a shows the original optical image. The RGB image is represented in a single grey scale matrix (Fig. 3b). Then a filter is applied to digitalize the image into black and white pixels (Fig. 3c). This is performed by setting a threshold level that defines which binary color is assigned. In Fig. 3d, a graphical representation of the BD spot locations and their areas is displayed in a window of size 438 $\mu \mathrm{m} \times 365 \mu \mathrm{m}$. Notice that the area of the circles represented in Fig. 3d is proportional to the discretized area of the spots. It is worth pointing out that the accuracy in the detection process is directly related to the resolution of the image, i.e. the smallest area we can detect is the area of a single marked pixel. For example, an image with dimensions 1920 px x 1080 px with a real width of $200 \mu \mathrm{m}$ have a scale of $0.104 \mu \mathrm{m} / \mathrm{px}$, which means that the size of a single pixel is $1.08 \cdot 10^{-2} \mu \mathrm{m}^{2}$ being the smallest area detectable in such image. In addition, in the analysis of a

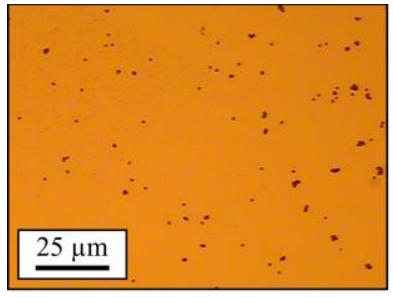

(a)

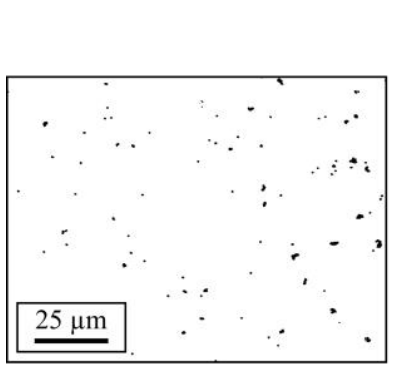

(c)

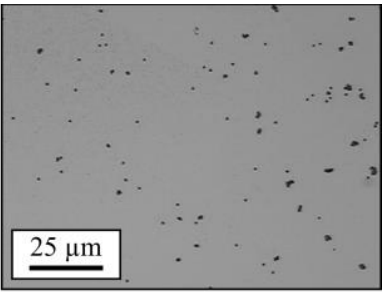

(b)

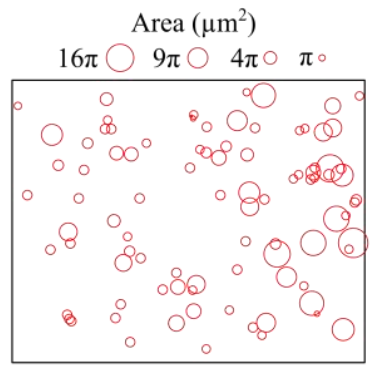

(d)
Fig. 3. (a) Optical image of a BD spot pattern. (b) Gray scale image of the image shown in (a). (c) Black and white image obtained after applying a binary filter. (d) Representation of the location and area of the black points shown in (c). The window dimension is $438 \mu \mathrm{m} \times 365 \mu \mathrm{m}$ and the size of the spots is proportional to the actual physical area.

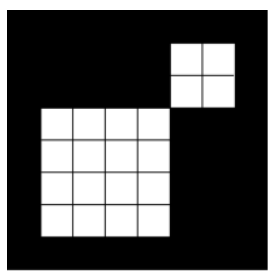

(a)

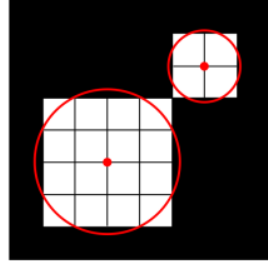

(b)

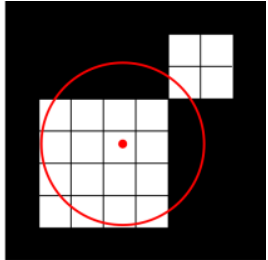

(c)
Fig. 4. (a) Schematic representation of a binary image. Spots obtained from the analysis using (b) 4-connected or (c) 8-connected pixels approach.

binary image, the superposition or connection of spots is a factor that needs to be considered [16]. For 2D images, pixels can be 4-connected, meaning that the pixels are connected if their edges touch, or 8-connected, meaning that pixels are connected if their edges or corners touch. In Fig. 4, a schematic representation of a binary image (Fig. 4a) considering both pixel arrangements (Fig. 4b-c) is illustrated. As can be seen, when the 4-connected approach is used (Fig. 4b), a total of 2 spots are detected as the pixels that only share a corner are considered as individual spots. However, when the 8 -connected approach is applied (Fig. 4c), those pixels are connected which results in the detection of a single structure. Notice that the location of the spots is the center of mass of the different regions detected as individual objects and that the total area of the objects is the same in both cases in spite of being the number of objects detected different as the spots that are separated in the 4-connected approach (Fig. 4b) appear merged into a single spot in the 8-connected approach (Fig. 4c). For the analysis performed in this work, the 8-connected approach was considered. Once the pixels are counted, the area of each spot is calculated using the direct expression: 


$$
\text { Spot size }=n \times \text { pixel size }
$$

where $n$ is the number of pixels and pixel size is the actual size of a single pixel. After obtaining the location and area of every BD spot, it is also important to correctly assess the possible edge effects associated with the size of the considered observation window (if different from the device area). This is a classical problem of spatial statistics that deserves a brief explanation [17]. Figure 5a shows a schematic representation of several objects with different areas distributed within the observation window (dashed square). As can be seen, some of the objects are affected by the window edge which performs like a kind of censoring effect. Two approaches can be followed: plus-sampling (Fig. 5b) or minus-sampling (Fig. 5c) [18] depending on whether the object is considered or not for the computation of areas, locations, and estimators. Plussampling introduces bias in favor of the largest objects while minus-sampling favors the smallest objects. As an example, in Fig. 6, both sampling approaches with different observation windows were considered for an experimental BD spot distribution. Figure 6a corresponds to the optical image of the damaged device. The location and areas of the spots are obtained and then the plus-sampling and minus-sampling approaches are applied using square observation windows with different areas. From this exercise, the ratio between the total damaged area (the total area of the spots) and the damaged area within the observation window can be compared (Fig. 6b). As expected, the ratio obtained for minus-sampling is smaller than for plus-sampling. The discrepancy between both methods is remarkable for small windows but reduces as the observation window increases. This means that the analysis of a failure site distribution using a very small observation window can be affected by the chosen counting method. This issue in combination with the minus-sampling approach was considered in this work.

\section{CORRELATION ANALYSIS}

In this Section, the summary statistics on the spatial distribution and size of multiple $\mathrm{BD}$ spots on the top $\mathrm{Pt}$ electrode and the correlation analysis between their locations and areas are discussed. As mentioned in Section II, the devices were ramped (RVS) or constant (CVS) voltage stressed. In case of a ramped voltage stress, all the spots are generated almost instantaneously. On the contrary, for CVS, the spots are generated progressively so that the time evolution of the degradation process can be recorded. For this reason, for RVS an optical image corresponding to the final situation can only be analyzed, whereas for CVS, a video showing the generation of spots is also available. In either case, final or intermediate state, the resulting distribution of $\mathrm{BD}$ spots can be analyzed using the methods of spatial statistics.

The spatial characterization of the spots is carried out using first (average intensity) and second order estimators (functional estimators). These estimators indicate whether the statistical distribution of spots agrees with a CSR process or not. The pair

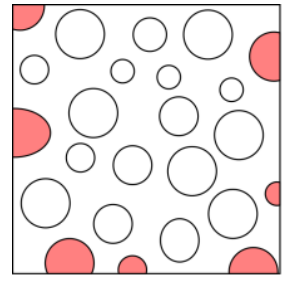

(a)

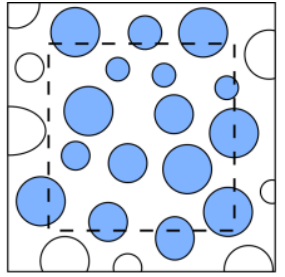

(b)

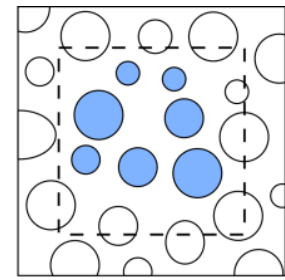

(c)
Fig. 5. (a) Schematic representation for the distribution of spots. The observation window performs as a kind of censoring effect. Spots analyzed using (b) a plus-sampling or (c) a minus-sampling approach.

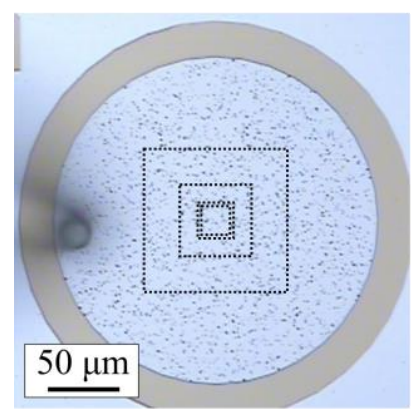

(a)

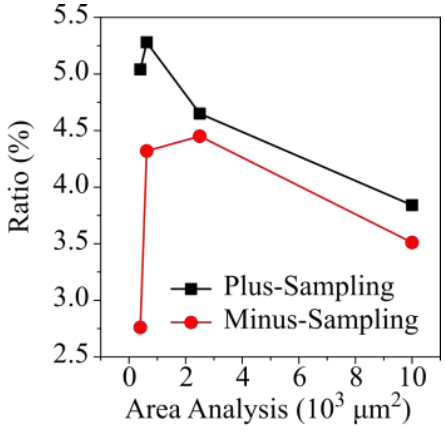

(b)
Fig. 6. (a) Optical image of a device showing multiple BD spots. (b) Ratio between the damaged area and the area of analysis for the different sampling approaches.

correlation function $g(r)$ is first used to investigate the $\mathrm{BD}$ point pattern. In this case, the distances between spots were normalized to the nominal lateral size of the device. $g(r)$ is related to the probability of finding a point at a distance $r$ from another point of the pattern [19]. The expected value for a CSR process is $g(r)=1$. Deviations from the unity value indicate accumulation $(g(r)>1)$ or inhibition $(g(r)<1)$ effects. In order to evaluate the connection between the spot locations and sizes, the mark correlation function $K(r)$ (not to be confused with Ripley's $K$ function) and the reverse conditional moments $E(r)$ and $V(r)$ are used [19]. For the computation of these latest estimators, distances are not normalized. The mark correlation function is a measure of the dependence between the locations of two points that are separated by a distance $r$ and the marks (or attributes) of these spots, in our case their areas. Following Stoyan [20], the mark correlation function $K(r)$ for a point process with numeric marks can be expressed as:

$$
K(r)=\frac{\mathbb{E}_{i j}\left[m_{i} \cdot m_{j}\right]}{\mu^{2}}
$$

where $\mathbb{E}_{\mathrm{ij}}$ denotes the conditional expectation given that there are a point $i$ and a point $j$ of the process separated by a distance r. $m_{i}$ and $m_{j}$ are the values of the marks attached to these two points. In the denominator of (2), $\mu$ is the mean value of the marks in the point process and it is used for normalizing the value of the function. In case of the mark correlation function, it is also possible to interpret the results in terms of accumulation or inhibition effects. If the areas of the spots are spatially uncorrelated, then $K(r)=1$. For positive correlation $K(r)<1$, which means that the spots separated by a distance $r$ 
have smaller area than expected, while a negative correlation $K(r)>1$ means the opposite [21]. $E(r)$ and $V(r)$ are the conditional mean and variance of the mark attached to a point that is located a distance $r$ from another point, respectively [22]. They can be expressed as:

$$
\begin{gathered}
E(r)=\mathbb{E}_{i j}\left[m_{i}\right] \\
V(r)=\mathbb{E}_{i j}\left[\left(m_{i}-E(r)\right)^{2}\right]
\end{gathered}
$$

$\mathbb{E}_{\mathrm{ij}}$ has the same meaning as before and $m_{i}$ denotes the mark attached to the point $i$. In this work, the attribute under analysis is always the area of the spot. Functions (3) and (4) also contribute to provide insight into the interrelationship between the spot locations and marks. If the points and marks are independent, then $E(r)$ and $V(r)$ should be constant (independent of $r$ ). In what follows, we apply this summary functions to different case studies.

\section{A. Square Area Capacitor}

In this first example, the case of a square area capacitor with an area of $750 \mu \mathrm{m} \times 750 \mu \mathrm{m}$ is investigated (see Fig. 7a). The spot pattern was generated using an RVS. In Fig. 7b, a graphical representation of the location of all the detected spots is shown. A total of 316 spots were counted which corresponds to an average intensity $\lambda=5.62 \cdot 10^{-4}$ spots $/ \mu \mathrm{m}^{2}$. The size of a single pixel is $3.04 \mu \mathrm{m}^{2}$. In Fig. 7c, a histogram for the radius of the spots is reported. It can be observed that the distribution exhibits a marked positive skewness with radius values ranging up to $5 \mu \mathrm{m}$. The experimental and theoretical $g(r)$ values are represented in Fig. 7d. Notice that the plot also includes the 95\% confidence band (shaded region). As can be seen, the experimental $g(r)$ fluctuates around the unity value which indicates that the distribution of failure sites is in principle compatible with a CSR process. Figure 7.e shows the experimental curve for the mark correlation function $K(r)$. Again, the shaded region corresponds to 50 Monte Carlo (MC) simulations. In this case, $K(r)$ indicates independence between positions and areas of the spots. In Fig. 7.f, the experimental $E(r)$ and $V(r)$ curves are shown. Notice that $E(r)$ fluctuates around the unity value, meaning that the spot locations and their areas are not connected. This agrees with the previous observations. However, $V(r)$ exhibits some peaks which might be indicative of a correlation between the location of the spots and their areas at certain scales. This example illustrates the importance of considering different estimators to assess a point pattern distribution and configure a better picture of what is going on. Since only $V(r)$ shows large deviations, it is hard to justify the existence of a correlation location/area for the BD spots. Both $g, K$, and $E$ functions seem to confirm that the point pattern follows a CSR process.

\section{B. Circular Area Capacitor}

In this second example, a circular area capacitor, in which a higher number of spots were generated, is analyzed. In Fig. 8a, the final distribution of BD spots obtained after an RVS is shown. The diameter of the device area is $1130 \mu \mathrm{m}$. A total of

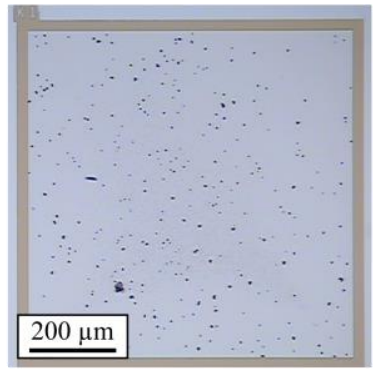

(a)

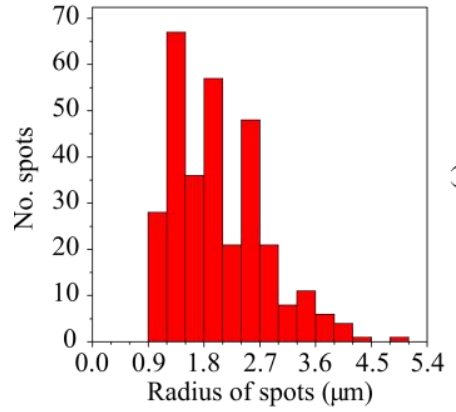

(c)

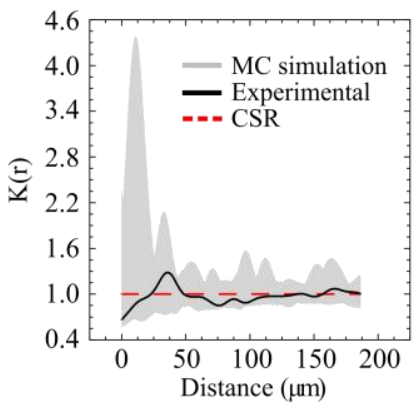

(e)

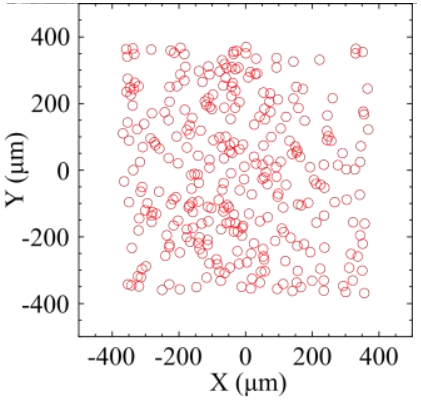

(b)

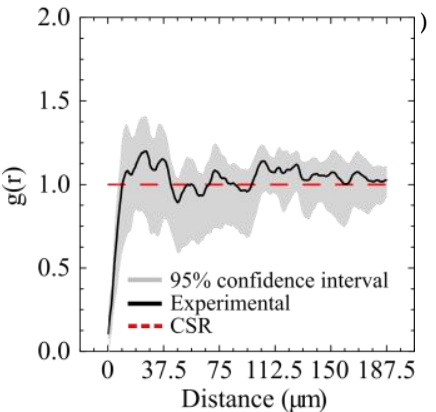

(d)

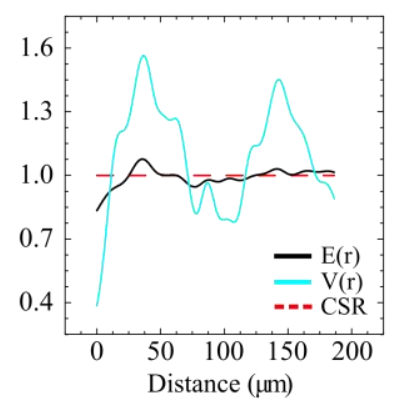

(f)
Fig. 7. (a) Photograph of a multiple BD spot distribution in a square area capacitor with size $750 \times 750 \mu \mathrm{m}$. (b) Location of points in the capacitor area. (c) Histogram plot of the spot area distribution. (d) Pair correlation function $g$ for the experimental data and for a CSR process. (e) Mark correlation function $K$ for the distribution of BD spots in the sample. (f) Experimental $E$ and $V$ functions and expected results for a CSR process.

1389 failure sites were detected which corresponds to an average intensity $\lambda=1.38 \cdot 10^{-3}$ spots $/ \mu \mathrm{m}^{2}$. The location of the detected spots is shown in Fig. 8b. In this case, the size of a pixel is $0.36 \mu \mathrm{m}^{2}$. In Fig. 8c, the histogram for the radius of the spots is reported. Notice that an accumulation of radius is observed for values ranging from $1.6 \mu \mathrm{m}$ to $3.2 \mu \mathrm{m}$ with a welldefined peak centered at $2.5 \mu \mathrm{m}$. In Fig. 8d, the experimental $g(r)$ function is shown. It is clearly above the unity value. This behavior is typical of some kind of accumulation effect. The origin of this deviation is clearly visible in the photograph of Fig. 8a, in which the largest area spots seem to concentrate in the central region of the device. If we now pay attention to the mark correlation function $K(r)$ in Fig. 8e, we can observe a correlation effect between the area and location of the spots as the experimental value exceeds the confidence band obtained after $50 \mathrm{MC}$ simulations. Again, this is consistent with the previous observation. Figure 8.f shows the estimated $E(r)$ and $V(r)$. Again, both curves depart from a CSR process. Large 


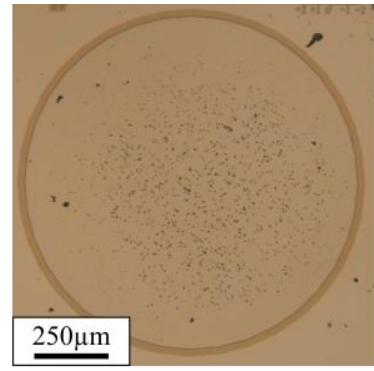

(a)

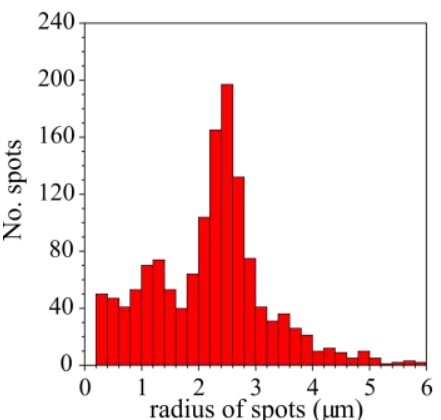

(c)

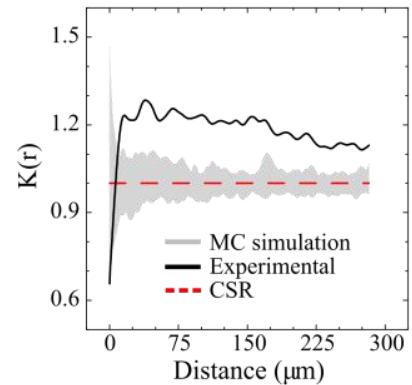

(e)

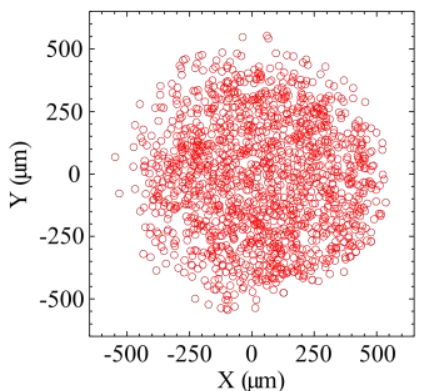

(b)

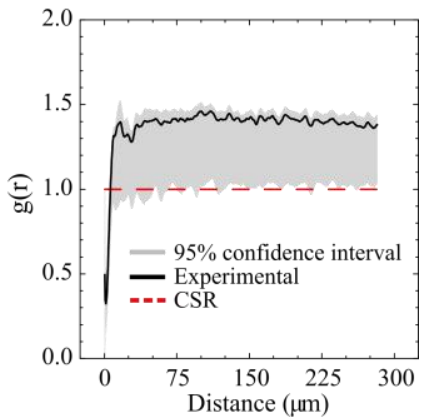

(d)

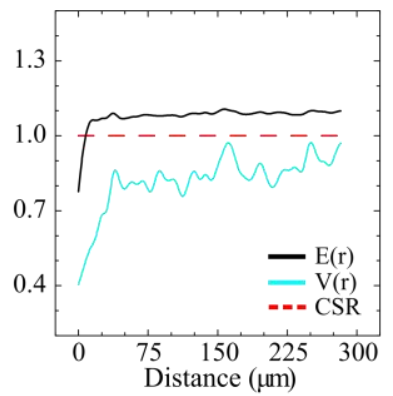

(f)
Fig. 8. (a) Photograph of a multiple BD spot distribution in a circular area capacitor with diameter of $1130 \mu \mathrm{m}$. (b) Location of points in the capacitor area. (c) Histogram for the spot radius. (d) Pair correlation function $g$ for the experimental data and for a CSR process. (e) Mark correlation function $K$ for the distribution of BD spots in the sample. (f) Experimental $E$ and $V$ functions and expected results for a CSR process.

deviations are observed close to the origin of coordinates but in this case the estimators are strongly influenced by the size of the spots and the anomalous short distance involved.

\section{EFFECTS OF SEVERE ELECTRICAL STRESS}

In the previous Section, it was shown that correlation effects between the location of the failure sites and their sizes can occur in large area MIM devices. This correlation can only be noticed if the number of spots is sufficiently large, which occurs after a long exposure to the degradation conditions. In this Section, we explore what happens if the device is further stressed and power dissipation effects become observable in the top metal electrode. We essentially focus the attention on the magnitude of the damage generated around the visible crater-like structure of the spots. In general, because of the series resistance effect, the pace at which the device degrades decreases with the number of spots, but at some point, a different kind of damage appears if the stress is not halted. Burning effects become evident in the surface of the device. In Fig. 9a, an optical image
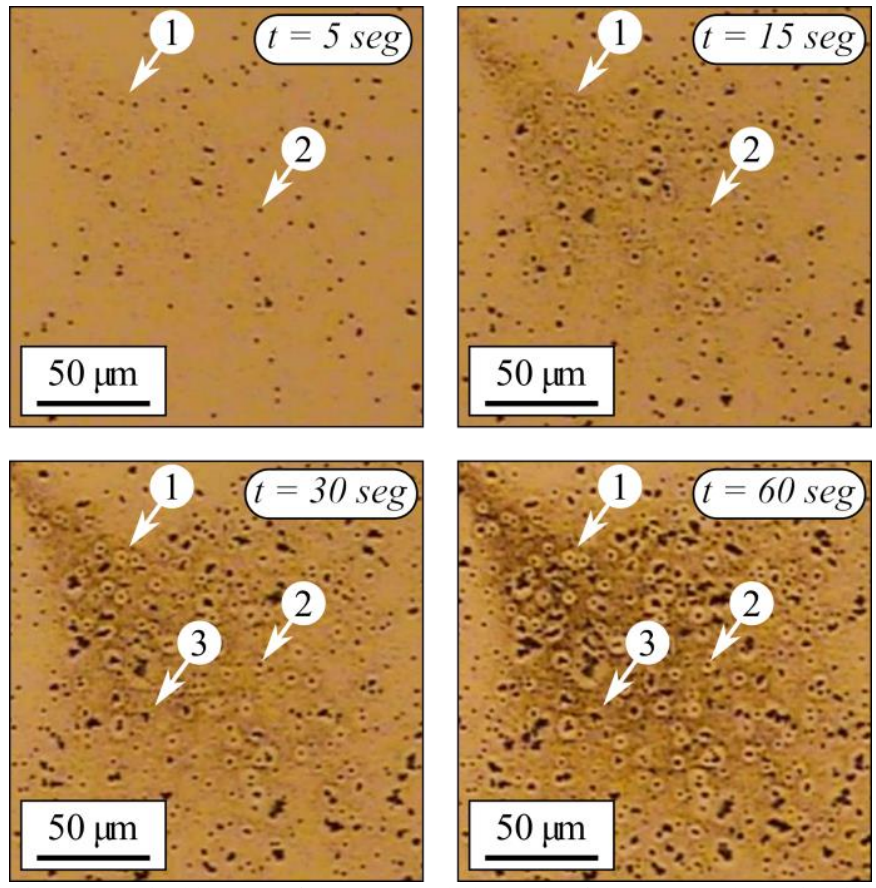

Fig. 10. Optical images of the top metal electrode of the sample shown in Fig. 9 a for different degradation times.

of a device that displays a burned region (darker area) is shown. This burning effect clearly arises from the voltage probe and directs toward the central region of the device. Around some BD spots, a small ring where the metal electrode is not burned is also visible. This is shown in Fig. 9b as well where a zoomed SEM image illustrates this situation. Figure 10 shows some specific frames of a video corresponding to the degradation of the device shown in Fig. 9a. The frames reveal that the rings are not visible until the area of the device around them starts burning. In other words, the rings were generated at the beginning of the stress (see arrows 1 and 2) but can only be observed in the last phase of degradation. This indicates that some structural modification occurred in the region close to the spot that reduces the surface current density around it preventing the appearance of the burned area. Something similar occurs for the spots that are generated in the already burned area (see arrow 3). Therefore, the thermal process associated with the generation of a BD site not only leaves a mark on the top metal electrode but also originates a damage beyond the spot itself.

In Figs. 11a-b, a CAFM image of a typical BD spot generated in the top metal electrode is shown. As can be observed, the spot has the usual crater-like structure. In order to analyze in detail if there is any kind of deformation of the metal layer around the spot, vertical and horizontal scanning profiles were defined in the image (Fig. 11b) and represented (Fig. 11c). The plot reveals that outside the spot (see the arrows), the Pt electrode remains completely flat meaning that the spot generation did not produce a hillock in the metal layer. The circular shape of the ring also discards a connection with the particular direction of the surface current lines responsible for the burning effects. A plausible hypothesis is that the physical changes in the dielectric region around the $\mathrm{CF}$ affect the current 


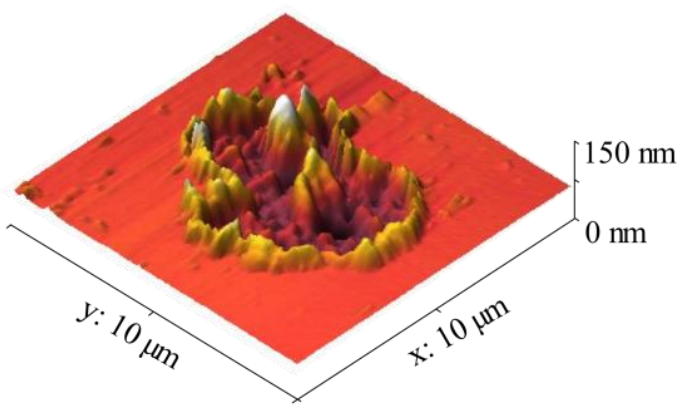

(a)

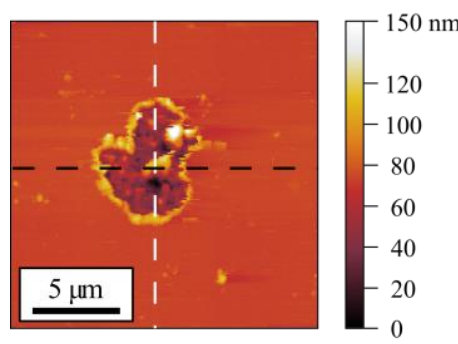

(b)

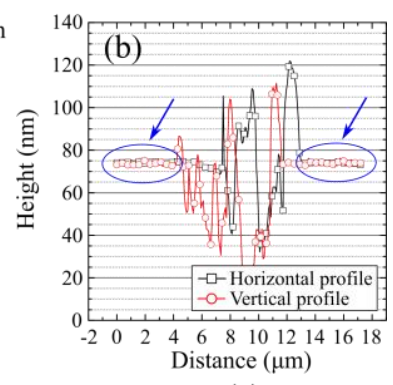

(c)
Fig. 11. AFM image of a spot generated in the top metal electrode in (a) 3D representation and (b) 2D representation. (c) Graphical representation of the profiles shown in (b).

distribution in the vicinity of the spot. In this regard, Cester $e t$ $a l$ reported a remarkable reduction of the conductance in a ring around the CF in the gate oxide of MOS transistors. The effect was ascribed to negative charge trapping and generation of oxide defects around the CF [23]. In more recent papers, Kumar et al reported modifications of the region surrounding the CFs generated in $\mathrm{Pt} / \mathrm{Hf} / \mathrm{HfO}_{\mathrm{x}} / \mathrm{Pt}$ [24] and $\mathrm{Pt} / \mathrm{TiN} / \mathrm{HfO}_{2} / \mathrm{Pt}$ [25] devices as well. Similar effects were described in Hoskins et al for $\mathrm{CFs}$ in $\mathrm{TiO}_{2}$-based MIM devices with Pt electrodes [26]. Experimental results seem to indicate a permanent change of the oxide properties in the vicinity of the failure site which does not generate a deformation of the metal electrode [27].

Figures 12a and $12 \mathrm{~b}$ show thermal images of a CF. In this case, a constant voltage was applied to the device and the power dissipated was registered using an infrared camera. While Fig. 12a shows the active BD spot and the voltage probes, Fig. 12b shows a detail of the damaged region. Notice that, although the area of the hot spot does not correspond to the real area of the failure site, it is clear that the temperature excess goe s beyond the core region of the CF. In order to evaluate the relative distribution of temperature produced by an active $\mathrm{CF}$, finiteelement simulations were carried out. Figure 12c shows a crosssection of the device assuming a filament temperature of $800 \mathrm{~K}$ and thermal conductivities $\lambda_{\mathrm{Pt}}=71 \mathrm{~W} \cdot \mathrm{K}^{-1} \cdot \mathrm{m}^{-1}$ and $\lambda_{\mathrm{HfO} 2}=0.95$ $\mathrm{W} \cdot \mathrm{K}^{-1} \cdot \mathrm{m}^{-1}[28]$. This plot can represent a situation previous to the occurrence of the microexplosion. As expected, the temperature increase extends farther into the dielectric than into the metal. This dissimilar temperature excess might be the reason behind the structural changes in the oxide layer and the reduced extension of the crater with respect to the surrounding ring. Interestingly, further information about the consequences of these anomalous regions can be achieved by analyzing the spatial distribution of the failure sites in severely damaged

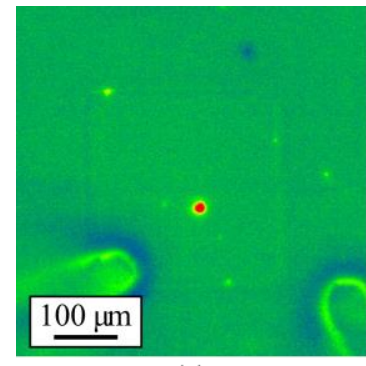

(a)

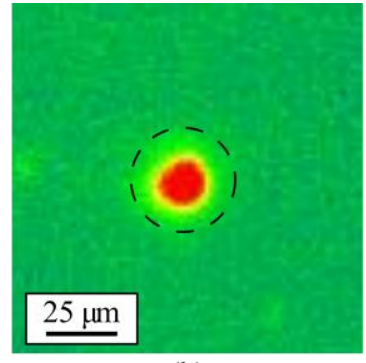

(b)

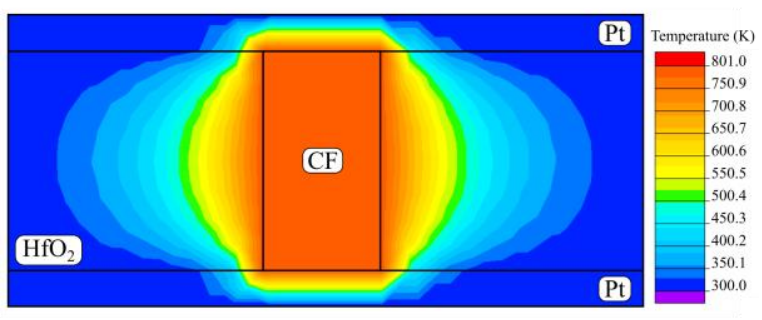

(c)

Fig. 12. (a) Thermal image of a square area capacitor with size $300 \mu \mathrm{m} \times 300$ $\mu \mathrm{m}$. (b) Zoom in thermal image of the hot spot detected in (a). (c) Thermal simulation of the distribution of temperature in a $\mathrm{Pt} / \mathrm{HfO}_{2} / \mathrm{Pt}$ device with a $\mathrm{CF}$ generated in the dielectric. The thermal conductivity considered are $\lambda_{\mathrm{Pt}}=71$ $\mathrm{W} \cdot \mathrm{K}^{-1} \cdot \mathrm{m}^{-1}$ and $\lambda_{\mathrm{HfO} 2}=0.95 \mathrm{~W} \cdot \mathrm{K}^{-1} \cdot \mathrm{m}^{-1}$. The external temperature is set to $300 \mathrm{~K}$ and the temperature of the $\mathrm{CF}$ is set to $800 \mathrm{~K}$.

devices. In this regard, Fig. 13 shows the case of a device with burning effects. In Fig. 13a the optical image of the capacitor with a diameter of $450 \mu \mathrm{m}$ is shown. The size of a pixel is 1.07 $\mu \mathrm{m}^{2}$. Figure 13b shows the location of $641 \mathrm{BD}$ spots, which corresponds to an average intensity $\lambda=4.03 \cdot 10^{-3} \mathrm{spots} / \mu \mathrm{m}^{2}$. The summary statistics for this point pattern is illustrated in Figs.13c-f. In Fig. 13c, the histogram for the size of the spots considered in the distribution is shown. Figure 13d shows the cumulative distribution function for the nearest-neighbour distance estimator $G(r)$ corresponding to the experimental distribution [9], [19]. The dashed line corresponds to a CSR process. The $95 \%$ confidence band is also included in the plot (shaded region). In order to analyze this result in detail it is important to consider the radius of the spots (Fig. 13c). In the $G$ function plot (Fig. 13d) an inhibition distance up to $8 \mu \mathrm{m}$ is observed as the experimental $G(r)$ takes values lower than those expected for a CSR distribution. This means that the generation of a spot in this region is less likely than for a Poisson distribution. In fact, $G(r)$ is zero for distances lower than $4 \mu \mathrm{m}$ meaning that there are no spots closer than $4 \mu \mathrm{m}$. Looking at the distribution of radius (Fig. 13c) and considering the accumulation of spots sizes ranging in between $1.1 \mu \mathrm{m}$ and 1.7 $\mu \mathrm{m}$, the expected inhibition distance would be $3.83 \mu \mathrm{m}$ which correspond to two spots with radius $1.4 \mu \mathrm{m}$ separated one pixel apart. Notice that the region where the inhibition is observed (Fig. 13d) is higher than the corresponding expected value. This confirms that there is an inhibition region around the spots where the generation of new failure sites is less likely. In Fig. $13 \mathrm{e}$, the experimental and theoretical $g(r)$ functions are illustrated. As can be seen, the location of all the spots is consistent with a CSR process as the unity value is inside the 95\% confidence band. Similarly, $K(r)$ in Fig. $13 f$ shows results compatible with independent location/area distributions. It 


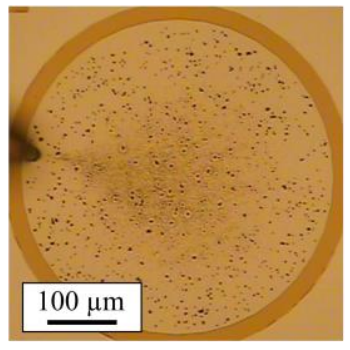

(a)

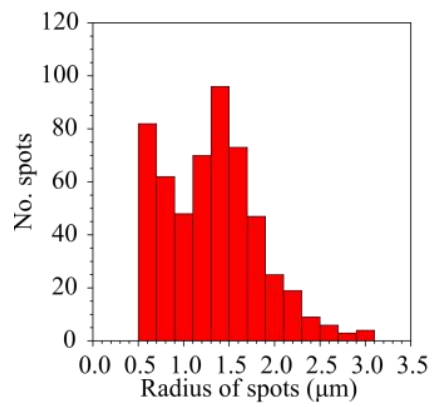

(c)

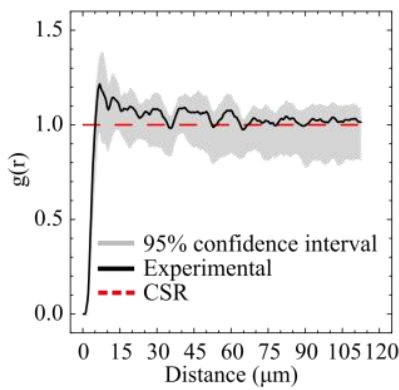

(e)

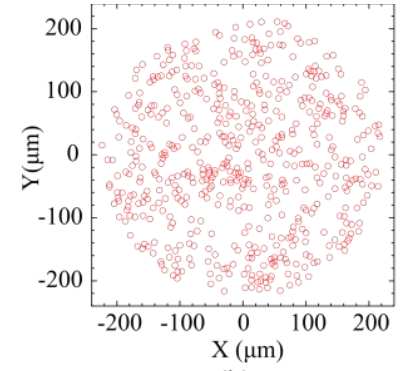

(b)

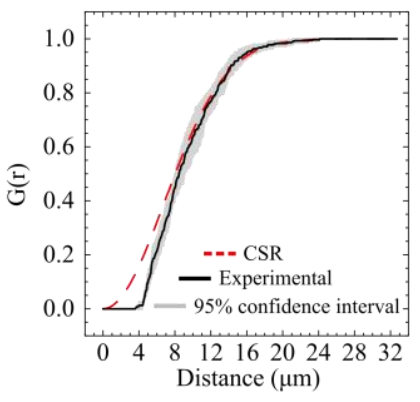

(d)

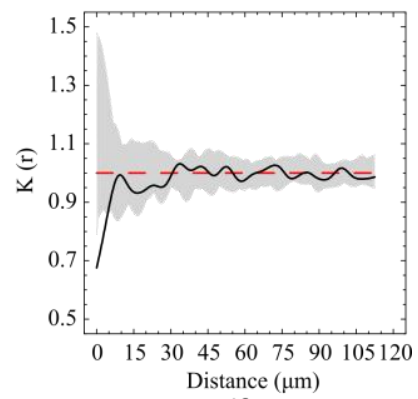

(f)
Fig. 13. (a) Photograph of a multiple BD spot distribution in a circular area capacitor with diameter of $450 \mu \mathrm{m}$. (b) Location of points in the capacitor area. (c) Histogram plot for the spot radius distribution. (d) Nearest-neighbour function $G$ for the experimental data and for a CSR process. (e) Pair correlation function $g$ for the experimental data and for a CSR process. (f) Mark correlation function $K$ for the distribution of BD spots in the sample.

seems that these estimators are not sensible enough to detect the inhibition region which is only observable in the short distance scale. This clearly indicates that caution should be exercised with a straightforward interpretation of the functional estimators.

\section{CONCLUSION}

In this paper, spatial statistical methods were applied to investigate the correlation between the location and size of catastrophic breakdown spots in MIM capacitors with high-K dielectric. The analysis revealed that for a low number of spots the distribution is consistent with a complete spatial randomness process. However, as the number of spots increases, correlation effects between the location and area become observable. The largest spots seem to concentrate in the central region of the device perhaps as a consequence of some kind of feedback effect resulting from the thermal effects and the current distribution over the top electrode. Inhibitory effects in the generation of spots were also detected in the last phase of degradation. To reveal these features, the separation between spots was investigated using specific estimators such as the nearest neighbour function. The observation of such inhibitory effects questions the hypothesis of complete randomness distribution in severely damaged devices.

\section{ACKNOWLEDGMENT}

The authors acknowledge support from MINECO, Spain (project TEC2017-84321-C4-4-R). JMG acknowledges the participation in the ASCENT project at CEA-Leti, France.

\section{REFERENCES}

[1] A. S. Oates, "Reliability issues for high-k gate dielectrics," in IEEE IEDM, Whasington, DC, USA, 2003, pp. 923-926, DOI: 10.1109/IEDM.2003.1269429.

[2] S. Lombardo, J. H. Stathis, B. P. Linder, K. L. Pey, F. Palumbo, and C. H. Tung, "Dielectric breakdown mechanisms in gate oxides," $J$. Appl. Phys., vol. 98, no. 12, 121301, 2005, DOI: 10.1063/1.2147714.

[3] T. Pompl, C. Engel, H. Wurzer, and M. Kerber, "Soft breakdown and hard breakdown in ultra-thin oxides," Microelectron. Reliab., vol. 41, no. 4, pp. 543-551, 2001, DOI: 10.1016/S0026-2714(00)00253-5.

[4] E. Y. Wu, J. H. Stathis, and L.-K. Han, "Ultra-thin oxide reliability for ULSI applications," Semicond. Sci. Technol., vol. 15, no. 5, pp. 425-435, 2000, DOI: 10.1088/0268-1242/15/5/301.

[5] M. A. Alam and R. K. Smith, "A phenomenological theory of correlated multiple soft-breakdown events in ultra-thin gate dielectrics," in IEEE IRPS, Dallas, TX, USA, 2003, pp. 406-411, DOI: 10.1109/RELPHY.2003.1197782.

[6] K. L. Pey, C. H. Tung, L. J. Tang, W. H. Lin, and M. K. Radhakrishnan, "Size difference in dielectric-breakdown-induced epitaxy in narrow n- and p-metal oxide semiconductor field effect transistors," Appl. Phys. Lett., vol. 83, no. 14, pp. 2940-2942, 2003, DOI: 10.1063/1.1616195.

[7] R. Ranjan, K. L. Pey, C. H. Tung, L. J. Tang, G. Groseneken, L. K. Bera and S. D. Gendt, "A comprehensive model for breakdown mechanism in $\mathrm{HfO}_{2} /$ high- $\kappa$ gate stacks," in IEEE IEDM, San Francisco, CA, USA, 2004, pp. 725-728, DOI: 10.11009/IEDM.2004.1419273.

[8] S. Chatterjee, Y. Kuo, J. Lu, J.-Y. Tewg, and P. Majhi, "Electrical reliability aspects of $\mathrm{HfO}_{2}$ high-k gate dielectrics with TaN metal gate electrodes under constant voltage stress," Microelectron. Reliab., vol. 46, no. 1, pp. 69-76, 2006, DOI: 10.1016/j.microrel.2005.02.004.

[9] X. Saura, J. Suñé, S. Monaghan, P. K. Hurley, and E. Miranda, "Analysis of the breakdown spot spatial distribution in $\mathrm{Pt} / \mathrm{HfO}_{2} / \mathrm{Pt}$ capacitors using nearest neighbor statistics," J. Appl. Phys., vol. 114, no. 15,154112 , 2013, DOI: $10.1063 / 1.4825321$.

[10] J. Muñoz-Gorriz, S. Monaghan, K. Cherkaoui, J. Suñé, P. K. Hurley, and E. Miranda, "Exploratory study and application of the angular wavelet analysis for assessing the spatial distribution of breakdown spots in $\mathrm{Pt} / \mathrm{HfO}_{2} / \mathrm{Pt}$ structures," J. Appl. Phys., vol. 122, no. 21, 215304, 2017, DOI: 10.1109/TDMR.2014.2369377.

[11] J. Muñoz-Gorriz, S. Monaghan, K. Cherkaoui, J. Suñé, P. K. Hurley, and E. Miranda, "Characterization of the Failure Site Distribution in MIM Devices Using Zoomed Wavelet Analysis," J. Electron. Mater., vol. 47, no. 9, pp. 5033-5038, 2018, DOI: 10.1007/s11664-0186298-2.

[12] E. Miranda, D. Jiménez, J. suñé, E. O'Connor, S. Monaghan, I. Povey, K. Cherkaoui, P. K. Hurley, "Nonhomogeneous spatial distribution of filamentary leakage current paths in circular area $\mathrm{Pt} / \mathrm{HfO}_{2} / \mathrm{Pt}$ capacitors," J. Vac. Sci. Technol. B Microelectron. Nanom. Struct., vol. 31, no. 1, 01A107, 2013, DOI: 10.1116/1.4768681.

[13] D. Illian, J., Penttinen, A. , Stoyan, H. and Stoyan, "The homogenous Poisson Process" in Statistical Analysis and Modelling of Spatial Point Patterns. Chichester, England: John Wiley \& Sons, 2008, pp. 57-98.

[14] A. Baddeley and R. Turner, "spatstat: An R Package for Analyzing Spatial Point Patterns," J. Stat. Softw., vol. 12, no. 6, 2005, DOI: 10.18637/jss.v012.i06.

[15] E. Miranda, M. Riccio, G. De Falco, J. Blasco, J. Suñé, and A. Irace, "Assessing the spatial correlation and conduction state of breakdown spot patterns in $\mathrm{Pt} / \mathrm{HfO}_{2} / \mathrm{Pt}$ structures using transient infrared thermography," J. Appl. Phys., vol. 115, no. 17, p. 174502, 2014, 
DOI: $10.1063 / 1.4874740$.

[16] L. He, X. Ren, Q. Gao, X. Zhao, B. Yao, and Y. Chao, "The connected-component labeling problem: A review of state-of-the-art algorithms," Pattern Recognit., vol. 70, pp. 25-43, 2017, DOI: 10.1016/j.patcog.2017.04.018.

[17] D. A. Griffith, "Correcting for Edge Effects in Spatial Statistical Analyses," in Advanced Spatial Statistics. Advanced Studies in Theoretical and Applied Econometrics, Dordrecht, Nederland: Springer, 1988, vol. 12, pp. 175-199, DOI: 10.1007/978-94-0092758-2_7.

[18] O. Barndorff-Nielsen, W. Kendall, M. N. M. Lieshout, A. Baddeley, C. Geyer, J. Moller, L. Vincent, I. Molchanov, I. Dryden and L. Saloff-Coste "Spatial Sampling and Censoring," in Stochastic Geometry: Likelihood and Computation, London: Chapman and Hall, 1999, pp. 37-78.

[19] A. Baddeley, E. Rubak, and R. Turner, Spatial point patterns : methodology and applications with $R$. Boca Raton, United States:CRC, 2015.

[20] D. Stoyan and H. Stoyan, Fractals, random shapes, and point fields : methods of geometrical statistics. Chichester, England: John Wiley \& Sons, 1994.

[21] K. Wälder and O. Wälder, "Analysing interaction effects in forests using the mark correlation function," iForest - Biogeosciences For., vol. 1, no. 1, pp. 34-38, 2008, DOI: 10.3832/ifor0449-0010034.

[22] M. Schlather, P. J. Ribeiro, and P. J. Diggle, "Detecting dependence between marks and locations of marked point processes," J. R. Stat. Soc. B, vol. 66, no. 1, pp. 79-93, 2004, DOI: 10.1046/j.13697412.2003.05343.x.

[23] A. Cester, A. Paccagnella, G. Ghidini, S. Deleonibus, and G. Guegan, "Collapse of MOSFET Drain Current After Soft Breakdown," IEEE Trans. Device Mater. Reliab., vol. 4, no. 1, pp. 63-72, 2004, DOI: 10.1109/TDMR.2003.820296.

[24] S. Kumar, Z. Wang, X. Huang, N. Kumari, N. Davila, J. P. Strachan, D. Vine, A. L. D. Kilcoyne, Y. Nishi and R. S. Williams, "Conduction Channel Formation and Dissolution Due to Oxygen Thermophoresis/Diffusion in Hafnium Oxide Memristors," ACS Nano, vol. 10, no. 12, pp. 11205-11210, 2016, DOI: 10.1021/acsnano.6b06275.

[25] S. Kumar, Z. Wang, X. Huang, N. Kumari, N. Davila, J. P. Strachan, D. Vine, A. L. D. Kilcoyne, Y. Nishi and R. S. Williams, "Oxygen migration during resistance switching and failure of hafnium oxide memristors," Appl. Phys. Lett., vol. 110, no. 10, 103503, 2017, DOI: 10.1063/1.4974535.

[26] B. D. Hoskins, G. C. Adams, E. Strelcov, N. Zhitenev, A. Kolmakov, D. B. Strukov and J. J. McClelland, "Stateful characterization of resistive switching $\mathrm{TiO} 2$ with electron beam induced currents," Nat. Commun., vol. 8, no. 1, 1972, 2017, DOI: 10.1038/s41467-01702116-9.

[27] C. H. Tung, K. L. Pey, L. J. Tang, M. K. Radhakrishnan, W. H. Lin, F. Palumbo and S. Lombardo, "Percolation path and dielectricbreakdown-induced-epitaxy evolution during ultrathin gate dielectric breakdown transient," Appl. Phys. Lett., vol. 83, no. 11, pp. 22232225, 2003, DOI: 10.1063/1.1611649.

[28] M. A. Panzer, M. Shandalov, J. A. Rowlette, Y. Oshima, Y. W. Chen, P. C. McIntyre, and K. E. Goodson, "Thermal properties of ultrathin hafnium oxide gate dielectric films," IEEE Electron Device Lett., vol. 30, no. 12, pp. 1269-1271, 2009, DOI: 10.1109/LED.2009.2032937. 